\title{
INVESTIGACIONES
}

\section{Liderazgo para la mejora en escuelas vulnerables: prácticas asociadas al cumplimiento de metas de aprendizaje*}

\author{
Leadership for school improvement in vulnerable schools: \\ leadership practices associated to goal achievement
}

\author{
Viola Espínola Hoffmann, ${ }^{a}$ Juan Ernesto Treviño, ${ }^{b}$ \\ Magdalena Guerrero Mena, ${ }^{c}$ José Martínez Argel ${ }^{d}$
}

aDirectora Magíster en Liderazgo y Gestión Educativa, Universidad Diego Portales Telf.: (56) 989207387. Correo electrónico: viola.espinola@ gmail.com

'Director Centro de Políticas Comparadas en Educación, Universidad Diego Portales

Telf.: (56) 977683540. Correo electrónico: trevino.ernesto@gmail.com

${ }^{c}$ Coordinadora de Extensión e Investigación del Magíster en Liderazgo y Gestión Educativa,

Universidad Diego Portales

Telf.: (56) 997453065. Correo electrónico: magdalena.guerrero@mail.udp.cl

dAnalista en Centro de Estudios del MINEDUC

Telf.: (56) 974738373. Correo electrónico: jose.martinez.argel@gmail.com

\section{RESUMEN}

La política de asignar a las escuelas una Subvención Escolar Preferencial (SEP) en función de los estudiantes de mayor vulnerabilidad introdujo metas de aprendizaje exigentes y cambios en las prácticas de liderazgo. El presente estudio tiene por objetivo identificar las prácticas de liderazgo de directivos y sostenedores que se asocian a las probabilidades de cumplir las metas, analizando la distribución de estas dentro y fuera de la escuela. La investigación utiliza una metodología mixta con una primera fase cuantitativa (encuestas a directivos, profesores y sostenedores) y luego cualitativa (entrevistas en 8 escuelas), en una muestra de 204 escuelas de la Región Metropolitana de la primera cohorte de SEP (2008-2012). Los resultados evidencian que la práctica directiva de observar aula y retroalimentar a docentes aumenta la probabilidad de cumplir la meta, mientras que las escuelas cuyos sostenedores centralizan la toma de decisiones limitando los espacios de autonomía de las escuelas, las disminuyen.

Palabras clave: liderazgo escolar, liderazgo intermedio, Subvención Escolar Preferencial (SEP), escuelas vulnerables, metas de aprendizaje.

\section{ABSTRACT}

The Preferencial School Subsidy involves additional funding for schools according to the number of vulnerable students; at the same time, it sets highly demanding learning goals and modifies existing leadership practices. This study aims to identify the leadership practices of school principals and of mid-level administrators associated to goal attainment through the analysis of the distribution of these practices inside and outside school. The study uses a mixed methodology, with a quantitative phase (questionnaires) and a qualitative phase (interviews in 8 schools), in a sample of 204 school of the first group that participated in the SEP program located in the Santiago, Región Metropolitana. Results show that the school administration practice of observing classes and providing feedback to teachers increases the probability of attaining the goal. On the other hand, schools in which mid-level

Artículo realizado en el marco del Proyecto FONDECYT nº 1120979 "Liderazgo para el mejoramiento educativo: Gestión del cambio para el logro de las metas de aprendizaje de los PM-SEP". 
administrators centralize the decision-making, provide limited spaces of autonomy to principals and teachers, reducing the probabilities of attaining the goal.

Key words: school leadership, mid-level administrators, Preferential School Subsidy, vulnerable schools, achievement goals.

\section{ANTECEDENTES}

En línea con las políticas de rendición de cuentas para mejorar la calidad de la instrucción en las escuelas, a partir del 2008 se implementó en Chile una política que apoya financieramente a las escuelas que atienden a estudiantes de nivel socio económico bajo mediante una Subvención Escolar Preferencial (SEP) para que estas mejoren sus resultados de aprendizaje. A las escuelas que adhieren a la SEP se les asigna una subvención adicional por alumno vulnerable ${ }^{1}$, lo que aumenta en un $50 \%$ a $60 \%$ el financiamiento estatal que reciben. Las escuelas deben firmar convenios de desempeño en los cuales se comprometen a cumplir metas de aprendizaje altamente exigentes. Las consecuencias de no cumplirlas ejercen una presión importante sobre las escuelas, puesto que estas arriesgan desde la intervención del Ministerio de Educación (MINEDUC) hasta el cierre definitivo.

El principal mecanismo de la SEP para transformar la entrega de recursos en más calidad instruccional es el Plan de Mejora (PM), que permite a las escuelas planificar y organizar las acciones para incrementar los aprendizajes de los estudiantes (MINEDUC, 2012).

En el PM las escuelas deben plantear sus metas de aprendizaje y comprometerse con acciones específicas para lograrlas en un periodo de 4 años. Las metas ponen el foco en lenguaje y matemática, su progreso es monitoreado a través de las evaluaciones anuales del Sistema de Información y Medición de la Calidad de la Educación (SIMCE) y han sido catalogadas como altamente demandantes (Elacqua, Mosqueira \& Santos, 2009). Si bien son las escuelas las que definen las metas a lograr, se les exige entre un $7 \%$ y $22 \%$ de aumento en los puntajes, siendo mayor la exigencia mientras más bajos sean estos antes de implementar los PM. Se ha planteado que el nivel de exigencia es demasiado alto, llegando en algunos casos a exigir aumentos de hasta 50 puntos, considerando que la experiencia internacional sugiere que aumentos de entre 5 y 10 puntos en este tipo de evaluaciones puede ser muy positivo (Elacqua et al., 2009).

Para la gestión de los PM y de los recursos para financiarlos, la política SEP introduce cambios importantes en las prácticas históricas de liderazgo del nivel intermedio (sostenedores) y de la escuela. En el pasado, las funciones de los sostenedores estaban focalizadas por ley en los aspectos administrativos y financieros de las escuelas (Espínola \& Silva, 2009; Espínola et al., 2010; Larraín, 2005; OCDE, 2010; Raczynski \& Salinas, 2006; Román, 2007). La SEP los lleva a asumir un papel central en los aspectos instruccionales y curriculares (González, González \& Galdames, 2015; Raczynski et al., 2013; Weinstein, Fuenzalida \& Muñoz, 2010). Al interior de la escuela, el equipo directivo, compuesto por un director, subdirector, inspector general y jefe técnico, distribuyeron tradicionalmente sus funciones de manera que

Para la SEP, los estudiantes vulnerables o prioritarios son aquellos para quienes “...la situación socio económica de sus hogares dificulta sus posibilidades de enfrentar el proceso educativo" y lograr que estos estudiantes alcancen aprendizajes equivalentes a los de sus compañeros más favorecidos tiene un costo mayor (Ley SEP, $\mathrm{N}^{\circ}$ 20.248). La vulnerabilidad de los estudiantes se define por el bajo nivel socioeconómico de las familias y el bajo nivel educativo de los padres. Por extensión, las escuelas que atienden a estudiantes vulnerables son denominadas escuelas vulnerables. 
los directores asumían las funciones más administrativas y los jefes técnicos los aspectos instruccionales (Núñez, Weinstein \& Muñoz, 2012). Con la SEP, los directores deben asumir funciones directamente relacionadas con la instrucción y la pedagogía (Espínola et al., 2010). Esta nueva asignación de funciones y responsabilidades plantea, de manera no explícita, un modelo de liderazgo sistémico según el cual tanto el sostenedor en el nivel intermedio como los directivos en las escuelas deben involucrarse en los aspectos instruccionales. La falta de definiciones claras respecto al rol que cada uno de los actores involucrados debe jugar genera tensión en la gestión de los PM-SEP (González et al., 2015).

Otra tensión se relaciona con las competencias de los actores para asumir las nuevas funciones de liderazgo. En el caso particular de las escuelas más vulnerables, hay evidencias de que las competencias técnicas de los sostenedores son insuficientes y a veces inexistentes (Raczynski, 2012). Entre los sostenedores municipales, 36\% de ellos no cuenta con personal en el área técnico-pedagógica y el personal que existe no siempre tiene las competencias profesionales o la iniciativa suficiente para encarar tareas técnicas (Laso, Albarrán \& Carrasco, 2012; MINEDUC, 2008).

Las evaluaciones preliminares de la SEP muestran tendencias positivas. Estudios sobre su impacto en el SIMCE evidencian que hay mejoras en el desempeño tanto en matemática como en lenguaje (Carrasco, Pérez \& Núñez, 2015), que el impacto de la SEP es mayor en escuelas con más altos porcentajes de estudiantes vulnerables (Valenzuela, Villarroel \& Villalobos, 2013) y que sus efectos son acumulativos, es decir, aumentan durante el desarrollo de los planes de mejora (Mizala \& Torche, 2013). También se han observado efectos positivos en las prácticas de los docentes, las que han puesto un foco mayor en los resultados de aprendizaje de los estudiantes (Weinstein et al., 2010), muestran una mayor frecuencia y profundidad de los procesos de planificación curricular (Raczynski, 2012) y una mayor socialización de las prácticas y conversaciones pedagógicas entre profesores (Weinstein et al., 2010). En cuanto a los directivos, se ha observado una creciente sistematicidad en la observación de aula (Raczynski et al., 2013).

Desde este marco de referencia, el estudio que aquí se presenta busca identificar las prácticas de liderazgo de directivos y de sostenedores que se asocian a las probabilidades de cumplir las metas definidas en los PM, con el fin de aportar con evidencias que permitan evaluar la política de gestión y liderazgo en la que se basa la SEP.

\section{MARCO CONCEPTUAL}

Para lograr este objetivo, se utilizaron dos marcos conceptuales inter-relacionados. Por una parte, las categorías claves del liderazgo efectivo propuestas por Leithwood (Louis et al., 2010), junto a una discusión sobre el liderazgo efectivo que se necesita para lograr la mejora en escuelas vulnerables sometidas a la presión de rendir cuentas (Ainscow, Muijs \& West, 2006; Chapman \& Harris, 2004; Fullan, 2005; Klar \& Brewer, 2013; Leithwood \& Strauss, 2008). Complementariamente, se utilizaron conceptos propios del liderazgo distribuido (Harris \& Spillane, 2008; Mayrowetz, 2008; Spillane et al., 2008) y del liderazgo sistémico para analizar las prácticas conjuntas de los directivos en las escuelas y de los sostenedores en el nivel intermedio (Burch \& Spillane, 2004; Epstein, Galindo \& Sheldon, 2011; Johnson \& Chrispeels, 2010; Sanders, 2012). La intersección entre estos marcos conceptuales permitió avanzar en la comprensión de la estructura de liderazgo y la distri- 
bución de prácticas directivas dentro y fuera de la escuela que se asocian a cumplimiento de metas y mejora de resultados de aprendizaje.

\subsection{LIDERAZGO EFECTIVO}

Diferentes estudios coinciden en señalar la importancia de una buena dirección para mejorar los aprendizajes en las escuelas (Louis et al., 2010; Robinson, 2007; Leithwood \& Riehl, 2005; Leithwood et al., 2004; Leithwood et al., 2006).

A partir del análisis de resultados de investigación en distintos contextos escolares, la contribución de varios investigadores ha dado lugar a un marco conceptual que organiza las prácticas efectivas en cuatro categorías clave para lograr un impacto sustancial en los aprendizajes (Leithwood, 2007; Leithwood \& Riehl, 2005; Leithwood et al., 2004). Las cuatro categorías clave descritas por Leithwood \& Jantzi (2008) son Mostrar dirección, Desarrollar personas, Rediseñar la organización y Gestionar la instrucción. Cada una de estas incluye un conjunto de prácticas que describen de manera más precisa el tipo de liderazgo que necesita desarrollar e implementar un director de escuela, en colaboración con otros miembros de su equipo directivo, para mejorar el desempeño (Anderson, 2010).

Las categorías clave han sido utilizadas para estudiar el liderazgo en escuelas que lograron revertir sus resultados (Leithwood, Harris \& Strauss, 2010), en escuelas sometidas a presión por mostrar resultados (Walker \& Ko, 2011) y en escuelas altamente vulnerables (Jacobson et al., 2005; Klar \& Brewer, 2013). En los casos de escuelas que revierten sus resultados de manera significativa, estudios han encontrado que todas las categorías habían tenido un peso en la mejora de los resultados, siendo la categoría Mostrar dirección la de mayor peso y Desarrollar personas la de menor peso (Leithwood \& Strauss, 2008).

\subsection{LIDERAZGO EN ESCUELAS VULNERABLES}

De importancia clave para las escuelas que adhieren a SEP, los efectos del liderazgo usualmente son mayores en establecimientos a los que asisten estudiantes vulnerables (Barber \& Mourshed, 2008; Leithwood et al., 2004; Leithwood et al., 2006; Leithwood et al., 2008; Mourshed, Chijioke \& Barber, 2010; Neumerski, 2012). Es más, no existen evidencias documentadas de escuelas de bajos resultados en condiciones difíciles (comunidades pobres, escasos recursos, geográficamente aisladas) que hayan logrado mejorar sin la intervención e influencia de un líder eficaz (Anderson, 2010).

\subsection{LIDERAZGO DISTRIBUIDO}

El liderazgo distribuido ha sido conceptualizado como una práctica conjunta que busca incidir sobre la escuela como un todo para lograr objetivos compartidos por la organización (Spillane et al., 2008)2 " "Comprende la identificación, adquisición, distribución, coordinación y uso de los recursos sociales, materiales y culturales que se necesitan para establecer las condiciones para la enseñanza y el aprendizaje" (Spillane et al., 2004, p. 11). El liderazgo distribuido involucra la acción coordinada de varios líderes, dentro y fuera de

\footnotetext{
La teoría del liderazgo distribuido ha sido construida con el aporte de muchos investigadores, empezando por Gronn (2002), Spillane, Halverson \& Diamond (2004), Mayrowetz (2008), Harris (2009), Leithwood et al. (2006).
} 
la escuela, para movilizar a la comunidad escolar a asumir las tareas necesarias para mejorar la instrucción, así como para asegurar los recursos para sustentar la transformación de la enseñanza y el aprendizaje (Harris \& Spillane, 2008; Mayrowetz, 2008). "Un meta análisis de varios estudios sobre liderazgo distribuido en escuelas mostró que éste tiene un impacto positivo en los profesores, en los directivos y en las escuelas como un todo" (Heikka, Waniganayake \& Hujala, 2012, p. 37) y que al vincular el liderazgo distribuido con los objetivos centrales de la enseñanza y el aprendizaje, la participación de todos los niveles de la administración y el apoyo de diferentes actores es clave.

\subsection{LIDERAZGO SISTÉMICO}

La mayor parte de la literatura sobre el liderazgo distribuido se refiere al liderazgo dentro de la escuela (Camburn et al., 2003; Firestone \& Martinez, 2007; Leithwood, 2007; MacBeath, 2005; Spillane, Camburn \& Pareja, 2007). Sin embargo, la distribución del liderazgo puede extenderse más allá de la escuela, hacia otras escuelas y hacia el nivel intermedio (Spillane, 1996). Según el liderazgo sistémico, los líderes confluyen desde sus diferentes posiciones en el sistema escolar, en prácticas para orientar, apoyar y dirigir a la escuela como un todo en el proceso de mejorar las prácticas instruccionales y fortalecer las capacidades colectivas (Harris \& Spillane, 2008).

En el marco de las políticas de rendición de cuentas, se producen cada vez con mayor frecuencia situaciones en las que el liderazgo se distribuye de manera sistémica, es decir, involucrando a líderes de distintos niveles de la estructura, por ejemplo, de la escuela y del distrito, del municipio, del Ministerio y también más allá de la estructura del sistema educativo para alcanzar a organizaciones no gubernamentales (Hallinger \& Heck, 2010; Spillane et al., 2004; Spillane at al., 2008). Esta consideración resulta crítica para la implementación de políticas a gran escala, como es el caso de la SEP. La perspectiva del liderazgo sistémico permite incorporar al sostenedor, como parte del esfuerzo colaborativo entre directivos dentro y fuera de la escuela para alcanzar las metas (Anderson, 2003; Leithwood et al., 2004).

\subsection{LIDERAZGO DE NIVEL INTERMEDIO}

La relativa dificultad que han tenido muchas reformas para llegar hasta las escuelas y abarcar al sistema educativo como un todo llevó a la conclusión de que los cambios significativos en las escuelas requieren de procesos de apoyo, de estructuras competentes y, de manera especial, de capacidades en los niveles intermedios (Sanders, 2012).

A partir de la década de los noventa, las políticas de rendición de cuentas experimentaron un cambio de foco según el cual se incluyó a los líderes distritales de manera que los directivos de las escuelas y los del distrito se involucraban de manera conjunta en la mejora y en la rendición de cuentas (Elmore, Abelmann \& Fuhrman, 1996). Es lo que sucede en el contexto de la SEP en Chile.

Esta tendencia se apoya en evidencias que muestran que las políticas de rendición de cuentas afectan de manera positiva el desarrollo organizacional cuando el sistema escolar como un todo, involucrando los liderazgos de los diferentes niveles, logra hacerse responsable por los resultados, más allá de la responsabilidad individual de los actores en cada una de las escuelas (Fullan, 2005). Más recientemente, evidencias de que los líderes 
distritales influyen en los resultados de las escuelas han despertado aún más el interés por el liderazgo de nivel intermedio (Anderson, 2003; Umekubo, Chrispeels \& Daly, 2013).

Se trata de una tendencia mundial la de darles a los líderes intermedios una injerencia mayor y más directa en los asuntos de las escuelas y en los aprendizajes de los estudiantes (Epstein et al., 2011; Hightower, 2002; Sanders, 2012).

Los líderes intermedios no generan las políticas y tampoco trabajan directamente con los profesores, sino que contribuyen a crear contextos coherentes y a garantizar las condiciones que las escuelas necesitan para implementar y sostener las reformas. Por su posición de articuladores de procesos de reforma entre el nivel central y las escuelas, los líderes intermedios son vistos por algunos como intermediarios que pueden movilizar recursos, información, conocimientos y competencias en apoyo de la implementación de los cambios en las escuelas (Burch \& Spillane, 2004). Orientar a las escuelas de manera que los profesores enseñen al máximo de su potencial y que los directivos sean capaces de liderarlos y de asegurar las condiciones para que esto suceda, requiere de un liderazgo intermedio altamente competente (Honig et al., 2010).

\subsection{LIDERAZGO Y REDES DE ESCUELAS}

Uno de los factores relacionados con la efectividad de los líderes intermedios es el tipo de relación que estos promueven entre las escuelas a su cargo. Para Muijs, West y Ainscow (2010) una red en educación se define como al menos dos organizaciones que trabajan de manera conjunta con un propósito compartido, por al menos un cierto tiempo. Esta definición deja abierta la posibilidad de colaboración horizontal o vertical entre escuelas y con el liderazgo intermedio, y proporciona un marco conceptual apropiado para analizar las prácticas de los sostenedores en relación a las escuelas que estos administran.

Las políticas de rendición de cuentas con un enfoque de redes potencian los buenos resultados cuando se logra asegurar un propósito colectivo liderado por los líderes intermedios, conexión entre las escuelas del distrito en función de la creación y fortalecimiento de sus capacidades, oportunidades de capacitación y desarrollo para los líderes de las escuelas y autoevaluación del progreso de la red de escuelas como un todo (Fullan, 2005).

Entre los beneficios de la colaboración entre escuelas se menciona la circulación de información útil para la toma de decisiones y la construcción horizontal de capacidades (Epstein et al., 2011). La colaboración permite la creación de comunidades de aprendizaje para la construcción de capacidades en todas las escuelas, y también en el liderazgo intermedio, para la mejora de los procesos instruccionales (Honig et al., 2010).

No obstante, aun cuando no exista esta colaboración, una red de escuelas reporta beneficios tales como las economías de escala que permiten, por ejemplo, compras de materiales al por mayor, capacitación docente a gran escala, compartir recursos, etc. (Hadfield, 2005). En el caso de la SEP se generan evidentes economías de escala al adscribir con una mayor cantidad de escuelas.

Por otra parte, la densidad de la red, determinada por la cantidad de escuelas bajo una misma administración, es un factor que incide en su efectividad. Una alta densidad —-mayor número de escuelas en la red — puede ser deseable en términos de profundizar la colaboración y maximizar las oportunidades para un aprendizaje colaborativo y un cambio cultural. Por otra parte, es importante guardar un equilibrio puesto que una red muy densa puede resultar muy difícil de gestionar (Muijs et al., 2010). 


\subsection{CENTRALIZACIÓN Y CONTROL VERSUS AUTONOMÍA}

El equilibrio adecuado entre centralización y autonomía es un factor clave a resolver en el marco de las políticas de rendición de cuentas. Por una parte, la presión para lograr resultados impacta en los líderes intermedios y estos tienden a aumentar el control sobre las escuelas y, por otra, las escuelas piden más espacios de autonomía para lograr la mejora en los resultados que se les piden (Johnson \& Chrispeels, 2010).

La literatura ofrece evidencias que sugieren que la decisión de dar más o menos autonomía a las escuelas debe considerar varios factores, tales como la capacidad profesional disponible en las escuelas, la calidad del liderazgo, la cantidad de estudiantes de bajo rendimiento y también la capacidad profesional disponible en el nivel intermedio. Las escuelas con buen nivel profesional entre sus directivos y profesores pueden hacer buen uso de los espacios de autonomía para lograr buenos resultados. En cambio, las escuelas con directivos y profesores débiles en cuanto a su capacidad profesional requieren de apoyo desde el nivel intermedio (Mourshed et al., 2010).

El equilibrio entre autonomía y control también debe considerar la trayectoria de aprendizaje de las escuelas. Mourshed et al., (2010) sostienen que las escuelas cuya trayectoria de rendimiento va de bueno a excelente, se benefician de una mayor autonomía. Por el contrario, cuando su rendimiento va de bajo a medio o de medio a razonable, estas se benefician de una mayor centralización y control. Cuando las trayectorias son decrecientes ha resultado más efectivo reducir la autonomía de las escuelas y entregarles planificaciones realizadas a nivel central, evaluaciones estandarizadas y otras medidas que requieren control desde el nivel intermedio (Dillon, 2011).

\section{METODOLOGÍA}

\subsection{MUESTRA}

La muestra utilizada en este estudio corresponde a 204 escuelas que imparten educación básica de la RM de Santiago, atienden a estudiantes vulnerables, adhirieron al programa SEP en el 2008 y mostraban una tendencia de estancamiento o baja en sus resultados de aprendizaje medidos por el SIMCE antes de implementar los PM-SEP.

Las escuelas se seleccionaron aleatoriamente a partir de bases de datos oficiales del MINEDUC, de un universo de 435 establecimientos de enseñanza básica. Para la selección de la muestra probabilística se siguió la técnica de muestreo aleatorio simple, utilizando varianza máxima, permitiendo un error máximo admisible de 5\%, con un 95\% de confianza.

\subsection{PROCEDIMIENTO DE LEVANTAMIENTO DE DATOS}

Los datos fueron recogidos mediante una encuesta elaborada en el marco de un proyecto FONDECYT (Fondo Nacional de Desarrollo Científico y Tecnológico) ${ }^{3}$ que indaga la relación entre prácticas de liderazgo y el cumplimiento de metas en el marco del programa SEP.

Proyecto FONDECYT n. ${ }^{\circ} 1120979$, "Liderazgo para el mejoramiento educativo: Gestión del cambio para el logro de las metas de aprendizaje de los PM-SEP”, 2012. 
Las preguntas (construidas en escalas Likert a partir de niveles de acuerdo y de frecuencia de prácticas en un rango de 1 a 5) fueron desarrolladas a partir de tres marcos conceptuales: i) las cuatro categorías claves del modelo de Leithwood (Leithwood \& Jantzi, 2008), ii) los estándares nacionales de competencias para los directores según el "Marco para la Buena Dirección" (MINEDUC, 2005) y iii) el "Modelo de gestión de calidad para sostenedores" (Fundación Chile, 2010).

La encuesta se aplicó de manera presencial a un miembro del equipo directivo del establecimiento involucrado en la implementación de los PM-SEP (70\% directores y 30\% jefes técnicos), 204 profesores(as) que hubieran trabajado también con metas SEP y 73 sostenedores de la misma muestra.

De manera complementaria, se realizaron entrevistas semi estructuradas en 8 de los 204 establecimientos, los que fueron seleccionados de acuerdo a su dependencia y trayectoria de puntajes SIMCE. En cada una de estas escuelas se entrevistó al sostenedor, al director y a tres docentes (40 entrevistas en total).

\subsection{PLAN DE ANÁLISIS}

El Plan de análisis se realizó en 4 etapas: en la primera etapa se cuantificó la variable dependiente denominada "cumplimiento de metas SEP", la que clasifica a los establecimientos según los que cumplieron las metas SEP y los que no las cumplieron, considerando los niveles de exigencia planteados por la SEP. En la segunda etapa se analizaron los datos de las encuestas para caracterizar las escuelas según variables relevantes para el cumplimiento de metas tales como cantidad de matrícula, promedio de alumnos por docente, número de alumnos prioritarios, entre otros. En la tercera etapa se clasificaron las prácticas de los directivos y de sostenedores en las 4 categorías clave de liderazgo exitoso. Se construyeron índices usando el método de componentes principales, a fin de reducir la dimensionalidad de los datos para realizar un análisis multivariado. En la cuarta etapa se llevó a cabo un análisis de regresión logística para estimar la probabilidad de cumplir con las metas en función de un conjunto de variables de liderazgo de directivos y sostenedores y de características de la escuela ${ }^{4}$.

Las estadísticas de las variables incluidas en esta investigación se presentan en la Tabla 1. El cumplimiento de meta SEP es la variable dependiente que toma valores dicotómicos de 0 o 1. Las demás variables son predictoras del cumplimiento de las metas SEP.

Tabla 1. Variables utilizadas en el análisis de cumplimiento de metas SEP

\begin{tabular}{|c|c|c|c|c|}
\hline Variable & Descripción & Prom & d.e. & $\mathrm{n}$ \\
\hline $\begin{array}{l}\text { Cumplimiento de meta } \\
\text { SEP }\end{array}$ & $\begin{array}{l}\text { Se clasifica con } 1 \text { a las escuelas que } \\
\text { consiguieron la meta y con } 0 \text { a las que no }\end{array}$ & 0.174 & 0.382 & 204 \\
\hline $\begin{array}{l}\text { Matrícula total en } \\
\text { enseñanza básica }\end{array}$ & $\begin{array}{l}\mathrm{N}^{\circ} \text { total de estudiantes de enseñanza } \\
\text { básica }\left(1^{\circ} \text { a } 8^{\circ}\right) \text { para el año } 2012\end{array}$ & 483 & 257 & 204 \\
\hline
\end{tabular}

Para realizar este análisis se utilizó la función Survey del Programa STATA 11.1, que permite producir resultados para datos de encuestas complejas, considerando el proceso de muestreo y pesaje de la misma. 


\begin{tabular}{|c|c|c|c|c|}
\hline $\begin{array}{l}\% \text { de asistencia promedio } \\
\text { en } 4^{\circ} \text { año básico }\end{array}$ & $\begin{array}{l}\text { Proporción de días asistidos respecto al } \\
\text { total de días hábiles en estudiantes de } 4^{\circ} \\
\text { año básico en el año } 2012 \text { por } \\
\text { establecimiento }\end{array}$ & 83.5 & 6.6 & 204 \\
\hline $\begin{array}{l}\mathrm{N}^{\circ} \text { de alumnos prioritarios } \\
\text { en el establecimiento para } \\
\text { el año } 2012\end{array}$ & $\begin{array}{l}\mathrm{N}^{\circ} \text { de alumnos prioritarios (beneficiarios } \\
\mathrm{SEP} \text { ) matriculados en el establecimiento }\end{array}$ & 335 & 191 & 204 \\
\hline \multicolumn{5}{|l|}{ 1. Muestra dirección } \\
\hline $\begin{array}{l}\text { 1.1. Equipo Directivo (ED) } \\
\text { promueve y controla las } \\
\text { metas de aprendizaje y } \\
\text { acciones comprometidas } \\
\text { en el PM-SEP }\end{array}$ & $\begin{array}{l}\text { Índice sumativo de variables relativas a } \\
\text { las prácticas de los directivos para } \\
\text { establecer, comunicar y promover entre } \\
\text { los docentes el logro de metas de } \\
\text { aprendizaje }\end{array}$ & 4.36 & 0.54 & 204 \\
\hline \multicolumn{5}{|l|}{ 2. Desarrolla personas } \\
\hline $\begin{array}{l}\text { 2.1. ED involucra a los } \\
\text { docentes en la planificación } \\
\text { y seguimiento de los PM- } \\
\text { SEP. }\end{array}$ & $\begin{array}{l}\text { Índice sumativo respecto al grado de } \\
\text { involucramiento del equipo de docentes } \\
\text { en el proceso de diagnóstico y } \\
\text { elaboración del PM-SEP }\end{array}$ & 4.31 & 0.57 & 204 \\
\hline \multicolumn{5}{|l|}{ 3. Gestiona la instrucción } \\
\hline $\begin{array}{l}\text { 3.1. ED se involucra en la } \\
\text { evaluación de los } \\
\text { aprendizajes logrados }\end{array}$ & $\begin{array}{l}\text { Índice sumativo del monitoreo de la } \\
\text { implementación curricular y las medidas } \\
\text { para construir capacidades. }\end{array}$ & 4.25 & 0.63 & 204 \\
\hline $\begin{array}{l}\text { 3.2. ED observa prácticas } \\
\text { de aula y proporciona } \\
\text { retroalimentación a los } \\
\text { docentes }\end{array}$ & $\begin{array}{l}\text { Índice sumativo de la práctica de } \\
\text { observación y retroalimentación de } \\
\text { prácticas de aula del ED }\end{array}$ & 4.41 & 0.68 & 204 \\
\hline $\begin{array}{lcr}\text { 3.3. Sostenedor } & \text { se } \\
\text { involucra en } & \text { el } \\
\text { mejoramiento } & \text { de } & \text { los } \\
\text { aprendizajes } & & \end{array}$ & $\begin{array}{l}\text { Índice sumativo de prácticas del } \\
\text { sostenedor involucrado en la mejora de } \\
\text { los aprendizajes, participando en el } \\
\text { diseño y seguimiento de estrategias de } \\
\text { mejora de resultados y eficiencia interna }\end{array}$ & 3.69 & 0.87 & 204 \\
\hline \multicolumn{5}{|l|}{ 4. Rediseña la organización } \\
\hline $\begin{array}{l}\text { 4.1. Sostenedor concentra } \\
\text { la responsabilidad final por } \\
\text { el PM-SEP }\end{array}$ & $\begin{array}{l}\text { El sostenedor concentra la } \\
\text { responsabilidad final por el PM-SEP }\end{array}$ & $0.729^{\mathrm{a}}$ & 0.44 & $159^{b}$ \\
\hline $\begin{array}{l}\text { 4.2. Sostenedor gestiona } \\
\text { recursos administrativos y } \\
\text { materiales para las } \\
\text { escuelas. }\end{array}$ & $\begin{array}{l}\text { Índice sumativo del grado de } \\
\text { involucramiento y apoyo del sostenedor } \\
\text { hacia acciones de mejora. }\end{array}$ & 3.77 & 0.96 & 204 \\
\hline
\end{tabular}

a Rango de variación entre 0 y 1, a diferencia de índices sumativos aquí considerados, que tienen un rango que va entre 1 y 5 . Variable originalmente en escala Likert (1 Muy en desacuerdo - 5 Muy de acuerdo), recodificada con "De acuerdo/Muy de acuerdo como 1 y el resto como 0.

b Este dato del sostenedor se replica en los establecimientos a los que correspondía el mismo sostenedor. 


\section{RESULTADOS}

\subsection{CUMPLIMIENTO DE LA META SEP}

Según se aprecia en la Tabla 2, solo 36 escuelas de la muestra cumplieron la meta $(17,6 \%)$ y 168 de ellas no lograron alcanzarla (82,5\%). Entre las que no lograron cumplir la meta, 54 escuelas $(26,5 \%)$ aumentaron su puntaje SIMCE en al menos 18 puntos $^{5}, 58(28,4 \%)$ escuelas aumentaron entre 1 y 17 puntos y 56 escuelas $(27,5 \%)$ disminuyeron su puntaje SIMCE durante la implementación del PM-SEP.

Tabla 2. Cantidad de escuelas según cumplimiento de metas: Variación del puntaje SIMCE Lenguaje $4^{\circ}$ Básico (2007- 2012)

\begin{tabular}{|l|c|c|}
\hline & Recuento & Porcentaje \\
\hline Cumple meta PM-SEP & 36 & $17,6 \%$ \\
\hline Aumenta mínimo de 18 puntos & 54 & $26,5 \%$ \\
\hline Aumenta entre 1 y 17 puntos & 58 & $28,4 \%$ \\
\hline Disminuye & 56 & $27,5 \%$ \\
\hline N & 204 & $100 \%$ \\
\hline
\end{tabular}

Si bien la mayoría de las escuelas de la muestra no cumplió la meta, un porcentaje importante de ellas (148 escuelas $=72,5 \%)$ logró aumentar sus puntajes. El promedio de aumento fue de 23,24 puntos.

Finalmente, los resultados que se muestran en la Tabla 3 evidencian que la mayor parte de las escuelas que logra la meta se encontraba en los rangos de más bajos puntajes SIMCE antes de ejecutar el PM-SEP, (menos de 250 puntos).

Tabla 3. Escuelas que cumplen la meta por nivel de exigencia

\begin{tabular}{|c|c|c|c|c|c|}
\hline Nivel & $\begin{array}{c}\text { Puntaje SIMCE } \\
\text { año } 2007\end{array}$ & $\begin{array}{l}\text { Meta } \\
\text { SEP }\end{array}$ & $\begin{array}{c}\mathrm{N}^{\mathrm{o}} \text { escuelas } \\
(2007)\end{array}$ & $\begin{array}{c}\mathrm{N}^{\mathrm{o}} \text { escuelas que } \\
\text { cumplen meta } 2012\end{array}$ & $\begin{array}{c}\% \text { escuelas que } \\
\text { cumplen meta } 2012\end{array}$ \\
\hline 1 & Menos de 220 & Aumentar un $22 \%$ & 33 & 9 & $25 \%$ \\
\hline 2 & Entre 220 y 250 & Aumentar un $14 \%$ & 109 & 22 & $61,1 \%$ \\
\hline 3 & Entre 250 y 280 & Aumentar un $12 \%$ & 53 & 2 & $5,6 \%$ \\
\hline 4 & Entre 280 y 300 & Aumentar un $7 \%$ & 9 & 3 & $8,3 \%$ \\
\hline Totales & & & 204 & 36 & $100 \%$ \\
\hline
\end{tabular}

Para las escuelas que justificadamente estimaban que no podrían cumplir la meta en los rangos sugeridos por la SEP, la política propuso un puntaje de aumento mínimo de 18 puntos (MINEDUC, Instructivo sobre metas de efectividad SEP, 2008). 
Esto podría explicarse en función de que las escuelas que iniciaron con desempeño más bajo tienen mayores posibilidades de mejora, asumiendo que están en una etapa de rendimientos crecientes.

\subsection{CARACTERÍSTICAS DE LAS ESCUELAS Y PRÁCTICAS DE LIDERAZGO}

Con el fin de identificar los factores de la escuela que vinculan el liderazgo del sostenedor y de los directivos con las probabilidades de alcanzar la meta, se utilizó un modelo de regresión logística (logit) para estimar la probabilidad de alcanzar las metas en función de un conjunto de variables de liderazgo y características de las escuelas. El modelo Logit establece la probabilidad de alcanzar las metas en las escuelas, la cual es condicional a un conjunto de variables predictoras relativas al liderazgo y a características de las escuelas. Dicha probabilidad es complementaria a la probabilidad de no alcanzar la meta dado ese mismo conjunto de covariables.

Para desarrollar este análisis se ajustaron los modelos incluyendo primeramente solo variables de caracterización de la escuela. Luego, se añadieron variables de liderazgo a partir de las respuestas de los directivos. Finalmente, se agregó la única variable de liderazgo del sostenedor que resultó estadísticamente significativa (Ver Tabla 4).

Tabla 4. Taxonomía de modelos Logit para estimar la probabilidad de alcanzar las metas PM-SEP en el periodo 2008-2012 en función de variables de liderazgo educativo y características de la escuela ${ }^{6}$

\begin{tabular}{|c|c|c|c|c|c|}
\hline & \multicolumn{5}{|c|}{ Modelos } \\
\hline & 1 & 2 & 3 & 4 & 5 \\
\hline \multicolumn{6}{|l|}{ Características de las escuelas (año 2012) } \\
\hline $\begin{array}{l}\text { Matrícula total en enseñanza básica en } \\
\text { el establecimiento (Número de } \\
\text { alumnos) }\end{array}$ & $\begin{array}{c}0.996 * * \\
(0.001)\end{array}$ & $\begin{array}{c}0.996 * * \\
(0.001)\end{array}$ & $\begin{array}{c}0.996 * * \\
(0.001)\end{array}$ & $\begin{array}{l}0.996^{*} \\
(0.001)\end{array}$ & $\begin{array}{l}0.997 \dagger \\
(0.001)\end{array}$ \\
\hline $\begin{array}{l}\text { Porcentaje de asistencia promedio en } \\
\text { cuarto año básico en el establecimiento }\end{array}$ & $\begin{array}{l}1.067 * * \\
(0.031)\end{array}$ & $\begin{array}{l}1.068^{* *} \\
(0.030)\end{array}$ & $\begin{array}{l}1.066^{* *} \\
(0.031)\end{array}$ & $\begin{array}{l}1.069^{* *} \\
(0.031)\end{array}$ & $\begin{array}{l}1.082 * * \\
(0.035)\end{array}$ \\
\hline $\begin{array}{l}\text { Número de alumnos prioritarios en el } \\
\text { establecimiento }\end{array}$ & $\begin{array}{c}1.001 \\
(0.001)\end{array}$ & $\begin{array}{c}1.001 \\
(0.001)\end{array}$ & $\begin{array}{c}1.001 \\
(0.001)\end{array}$ & $\begin{array}{c}1.001 \\
(0.001)\end{array}$ & $\begin{array}{c}1.000 \\
(0.001)\end{array}$ \\
\hline \multicolumn{6}{|l|}{ Liderazgo Directivo } \\
\hline \multicolumn{6}{|l|}{ Muestra dirección } \\
\hline $\begin{array}{l}\text { Equipo Directivo promueve y controla } \\
\text { las metas de aprendizaje y acciones } \\
\text { comprometidas en el PM-SEP }\end{array}$ & & $\begin{array}{c}1.255 \\
(0.745)\end{array}$ & $\begin{array}{c}1.073 \\
(0.658)\end{array}$ & $\begin{array}{c}0.847 \\
(0.599)\end{array}$ & $\begin{array}{c}1.249 \\
(0.886)\end{array}$ \\
\hline
\end{tabular}

6 Coeficientes expresados en odds ratio. Errores estándares robustos entre paréntesis. Modelos I-IV ajustados por peso muestral para 204 casos. Modelo V ajustado por peso muestral para 159 casos.

$* \mathrm{p}<0.01 * * \mathrm{p}<0.05{ }^{\dagger} \mathrm{p}<0.1$ 
Estudios Pedagógicos XLIII, N $^{\circ}$ 1: 87-106, 2017

LIDERAZGO PARA LA MEJORA EN ESCUELAS VULNERABLES: PRÁCTICAS ASOCIADAS AL CUMPLIMIENTO DE METAS DE APRENDIZAJE

\begin{tabular}{|c|c|c|c|c|c|}
\hline \multicolumn{6}{|l|}{ Desarrolla personas } \\
\hline $\begin{array}{l}\text { Equipo Directivo involucra a los } \\
\text { docentes en la planificación } \\
\text { seguimiento de los PM-SEP }\end{array}$ & & $\begin{array}{c}0.758 \\
(0.373)\end{array}$ & $\begin{array}{c}0.685 \\
(0.351)\end{array}$ & $\begin{array}{c}0.757 \\
(0.453)\end{array}$ & $\begin{array}{c}0.561 \\
(0.362)\end{array}$ \\
\hline \multicolumn{6}{|l|}{ Gestiona la Instrucción } \\
\hline $\begin{array}{l}\text { Equipo Directivo observa prácticas de } \\
\text { aula y proporciona retroalimentación a } \\
\text { los docentes }\end{array}$ & & & $\begin{array}{c}1.990 * * \\
(0.619)\end{array}$ & $\begin{array}{c}2.150^{* *} \\
(0.759)\end{array}$ & $\begin{array}{c}2.618^{* *} \\
(1.213)\end{array}$ \\
\hline $\begin{array}{l}\text { Equipo Directivo se involucra en la } \\
\text { evaluación de los aprendizajes logrados }\end{array}$ & & & $\begin{array}{c}0.999 \\
(0.288)\end{array}$ & $\begin{array}{c}0.972 \\
(0.286)\end{array}$ & $\begin{array}{c}0.826 \\
(0.333)\end{array}$ \\
\hline $\begin{array}{l}\text { Sostenedor se involucra en el } \\
\text { mejoramiento de los aprendizajes }\end{array}$ & & & & $\begin{array}{l}2.066 \dagger \\
(0.852)\end{array}$ & $\begin{array}{c}1.372 \\
(0.687)\end{array}$ \\
\hline \multicolumn{6}{|l|}{ Rediseña la organización } \\
\hline $\begin{array}{lll}\text { Sostenedor } & \text { gestiona } & \text { recursos } \\
\text { administrativos } & \text { y materiales } & \text { para las } \\
\text { escuelas } & & \\
\end{array}$ & & & & $\begin{array}{c}0.632 \\
(0.225)\end{array}$ & $\begin{array}{c}0.920 \\
(0.456)\end{array}$ \\
\hline \multicolumn{6}{|l|}{ Liderazgo del Sostenedor } \\
\hline \multicolumn{6}{|l|}{ Rediseña la organización } \\
\hline $\begin{array}{l}\text { Sostenedor concentra responsabilidad } \\
\text { final por el PM }\end{array}$ & & & & & $\begin{array}{l}0.239 * \\
(0.115)\end{array}$ \\
\hline Intercepto & $\begin{array}{c}0.003 * * \\
(0.007)\end{array}$ & $\begin{array}{c}0.003 * * \\
(0.009) \\
\end{array}$ & $\begin{array}{l}0.001 * \\
(0.001)\end{array}$ & $\begin{array}{l}0.000 * \\
(0.001) \\
\end{array}$ & $\begin{array}{l}0.000^{*} \\
(0.000)\end{array}$ \\
\hline Prob $>\mathrm{Chi}^{2}$ & 0.0123 & 0.0540 & 0.0091 & 0.0090 & 0.0001 \\
\hline Pseudo $\mathrm{R}^{2}$ & 0.0522 & 0.0540 & 0.0761 & 0.0965 & 0.1455 \\
\hline Log pseudolikelihood & -180.410 & -180.084 & -175.874 & -171.983 & -161.022 \\
\hline $\mathrm{N}$ & 204 & 204 & 204 & 204 & 159 \\
\hline
\end{tabular}

Según se aprecia en la Tabla 4, los resultados muestran que la matrícula de educación básica y el porcentaje de asistencia en $4^{\circ}$ básico condicionan de manera significativa las probabilidades de cumplir las metas en todos los modelos. Por el contrario, el número de alumnos prioritarios en el establecimiento es una variable que no guarda relación con las probabilidades de cumplir las metas. Este dato cobra una mayor relevancia considerando que es uno de los criterios que utiliza la política SEP para asignar los recursos a las escuelas.

En el caso de la matrícula en la enseñanza básica, por cada alumno adicional matriculado, las probabilidades de cumplir las metas disminuyen en un $0.4 \%$ (modelos 1 a 4) y en el caso de la asistencia, las escuelas con mayor asistencia promedio tienen mayores probabilidades de alcanzar las metas, las que por cada punto adicional, aumentan en un rango entre 6,6\% y $8,2 \%$ (modelos 1 al 5).

\subsection{PRÁCTICAS DE LIDERAZGO SEGÚN CUMPLIMIENTO DE LAS METAS}

Según el modelo 5 de la Tabla 4, solamente una práctica de los directores dentro de la escuela, "observa aula y retroalimenta", está asociada de manera positiva al cumplimiento de 
las metas SEP; es estadísticamente significativa en todos los modelos testeados (modelos 3 , 4 y 5). Por otro lado, entre las prácticas de los sostenedores fuera de la escuela se encontró una asociación negativa entre la práctica descrita como "el sostenedor concentra la responsabilidad final por el cumplimiento del PM", lo que implica que esta práctica disminuye la probabilidad de alcanzar las metas generadas a partir de la SEP.

El que solo un índice de liderazgo dentro de la escuela y uno fuera de la escuela hayan mostrado estar relacionados con la probabilidad de cumplir las metas no resulta extraño, dado que la relación entre liderazgo y resultados de aprendizaje es una relación indirecta, que está mediatizada por factores del docente y de la escuela (Anderson, 2010).

Dada la metodología de análisis utilizada, las prácticas directivas con foco en el aula constituyen el indicador más robusto para predecir significativamente el cumplimiento de la meta. Aquellas variables que no resultaron significativas ejercen la función de control estadístico, lo que enfatiza la robustez de la asociación entre la observación y retroalimentación de prácticas docentes y el cumplimiento de la meta SEP.

\section{Prácticas Directivas en la escuela}

Los resultados muestran que el incremento en una unidad en el índice de observación de aula y retroalimentación de prácticas docentes por parte del equipo directivo se asocia a una probabilidad de 2 a 2,6 veces mayor de alcanzar la meta.

Con el fin de indagar qué entienden los actores por "observa aula y retroalimenta", en la fase cualitativa del estudio se les preguntó por esta práctica. Según la describen los profesores de escuelas que cumplen las metas SEP, se trataría de una práctica más bien informal y asistemática aludiendo a directores que muestran interés y preocupación por los aspectos instruccionales haciéndose presentes en la sala de clases, preguntando por las planificaciones, las evaluaciones, la lista de asistencia, el libro de clases, y haciendo ocasionales comentarios informales sobre lo observado. No se trataría de observaciones en base a instrumentos estandarizados, registros rigurosos, cuantificación de actividades u otro procedimiento objetivo, cuantificable y comparable de observación de clases. Las siguientes respuestas de profesores reflejan esta percepción.

"El director es de los que pasa sala por sala todos los días, sí, y te viene a contar los niños de la clase o revisar la asistencia o viene como a buscar algo y en realidad tú sabes que viene a mirar la sala. Es como bien presente... de repente se acerca y te dice "mira puedes hacer esto...". (Profesor).

"El director visita sala y... ve las debilidades que hay, las debilidades profesionales y va de alguna manera tomando decisiones a partir del conocimiento que tiene del desarrollo de clases... es un director en ese aspecto en la gestión pedagógica muy bueno". (Profesor).

Las respuestas reflejan también un cambio en la distribución de tareas de liderazgo dentro de la escuela entre los directores y los jefes técnicos. En el marco de la SEP, los directores asumen tareas relacionadas con la instrucción y la supervisión de aula que antes estaban exclusivamente en manos de los jefes técnicos, percibiéndose poca claridad en la distribución de funciones entre ambos directivos. Como lo expresan un director y un profesor de escuelas que cumplen las metas: 
"La parte técnica pedagógica estaba netamente abocada a lo pedagógico, pero ahora uno está inserto dentro de lo pedagógico, porque uno (como director) también tiene que hacer acompañamiento al aula". (Director).

"El director y el feje de UTP van por todas las salas". (Profesor).

Estas respuestas reflejan un cambio en la histórica distribución de tareas entre el director y el jefe técnico en las escuelas, mostrando que hay una separación menos clara y una menor distinción entre ellas. Muestran que los directores están incursionando en funciones históricas del jefe técnico.

\section{Prácticas del Sostenedor}

Respecto a las prácticas de liderazgo de los sostenedores, cuando ellos mismos perciben que concentran la responsabilidad principal por la gestión de los PM, disminuyen las probabilidades de alcanzar las metas. Los resultados muestran que los establecimientos en los que el sostenedor se declara como el responsable por la gestión de los PM por sobre los directores de las escuelas, tienen solamente un $23,9 \%$ de probabilidades de alcanzar las metas.

Los análisis cualitativos mostraron que los sostenedores más efectivos — cuyas escuelas cumplen la meta- se muestran más inclinados a descentralizar las decisiones relacionadas con la gestión del PM y el control sobre el presupuesto y recursos necesarios para su ejecución, entregando autonomía a las escuelas para tomar decisiones y flexibilidad para gestionar los PM.

"Ese establecimiento ha tenido la particularidad de organizar muy bien los recursos y ser muy asertivos ese equipo, liderado por el director, para la utilización de recursos, porque yo diría que apuntó de forma muy efectiva en la mejora de aprendizajes, recursos humanos, recursos tecnológicos, recursos para fortalecer asignaturas, pertinentes en perfeccionamientos de sus profesores". (Sostenedor).

"El Director reconoció que la autonomía que nosotros les dábamos permitía obviamente desarrollar en forma mucho más eficaz o efectiva las propuestas que ellos tenían". (Sostenedor).

Asimismo, el análisis cualitativo mostró que los sostenedores efectivos otorgan más espacios de participación a las escuelas y muestran una alta valoración por la participación de todos los actores de la comunidad educativa durante la ejecución del PM-SEP.

"El equipo directivo tuvo una alta participación en el diagnóstico, porque nosotros no queríamos imponer nada y también (participó) el profesorado. Ellos han tenido opciones de poder opinar y poder estar a caballo de lo que se necesita". (Sostenedor). "Había un reconocimiento del trabajo en red, participativo, guardando por supuesto las particularidades de cada realidad de establecimiento". (Sostenedor).

\section{Densidad de la Red}

Por otra parte, los datos muestran que cuanto mayor es el número de escuelas que depende del sostenedor, menor es el porcentaje de escuelas que cumplen las metas SEP. Es decir, a mayor densidad de la red, menor es la probabilidad de alcanzar las metas. 
Tabla 5. Número de escuelas por sostenedor y cumplimiento de metas $\mathrm{SEP}^{7}$

\begin{tabular}{|l|c|c|c|c|c|c|}
\hline $\begin{array}{c}\text { Tamaño } \\
\text { de la red }\end{array}$ & $\begin{array}{c}\mathrm{N}^{\circ} \text { de } \\
\text { sostenedores }\end{array}$ & $\begin{array}{c}\% \text { respecto } \\
\text { al total de } \\
\text { sostenedores }\end{array}$ & $\begin{array}{c}\mathrm{N}^{\circ} \text { de } \\
\text { escuelas a } \\
\text { cargo de } \\
\text { cada } \\
\text { sostenedor }\end{array}$ & $\begin{array}{c}\mathrm{N}^{\circ} \text { de } \\
\text { escuelas } \\
\text { susceptibles } \\
\text { de adherir a } \\
\text { SEP en 2007 }\end{array}$ & $\begin{array}{c}\mathrm{N}^{\circ} \text { de } \\
\text { escuelas que } \\
\text { cumplen las } \\
\text { metas por } \\
\text { sostenedor }\end{array}$ & $\begin{array}{c}\% \text { de } \\
\text { escuelas que } \\
\text { cumplen las } \\
\text { metas por } \\
\text { sostenedor }\end{array}$ \\
\hline 1 escuela & 25 & $28,10 \%$ & 25 & 25 & 6 & $24,0 \%$ \\
\hline $2-5$ escuelas & 20 & $22,47 \%$ & 47 & 39 & 6 & $15,4 \%$ \\
\hline $6-20$ escuelas & 35 & $39,33 \%$ & 449 & 350 & 57 & $16,3 \%$ \\
\hline $21-50$ escuelas & 9 & $10,11 \%$ & 282 & 209 & 23 & $11 \%$ \\
\hline Total & 89 & $100 \%$ & 803 & 623 & 92 & $14,7 \%$ \\
\hline
\end{tabular}

Como se ve en la Tabla 5, los sostenedores que administran una sola escuela son los que tienen mayores probabilidades de cumplir las metas. En el caso de los sostenedores que tienen más de 20 establecimientos, solamente el $11 \%$ de estos cumplen las metas.

Lo óptimo es que un sostenedor administre una sola escuela, lo que posiblemente permite un conocimiento y cercanía mayor con la escuela, una supervisión o apoyo más directo. Entre 2 y 20 escuelas es una densidad adecuada y más de 20 resulta poco recomendable desde la perspectiva de la calidad de la supervisión y apoyo que el sostenedor puede prestar a su red de escuelas.

En este contexto, los sostenedores de escuelas que cumplen las metas hacen referencia a la importancia de la cercanía y conocimiento de cada escuela.

"Entonces, esto también nos ha ayudado a unirnos más con los colegios, poder marchar juntos, que vean al sostenedor no como una cosa externa, así de que viene del cielo, sino que en conjunto hemos ido trabajando con ellos, y eso yo lo valoro bastante porque permite que haya una mancomunión entre el sostenedor y los establecimientos". (Sostenedor).

"Que sientan que realmente tienen voz y voto también, en el decir las cosas y eso yo creo que es una contribución, para que uno como sostenedor pueda captar el ambiente. Conversar con los profesores, ver qué están haciendo, cómo lo están haciendo y qué dificultades tienen, eso yo creo que es un aporte...". (Sostenedor).

Asimismo, el tamaño de la red debe hacer posible la colaboración entre escuelas.

“... se están haciendo grupos de colegios en que se intercambian experiencias. Entonces, el colegio cuenta sus experiencias, después otro cuenta las de él, y así se van desarrollando conclusiones, y algunos se prestan ayuda. Por ejemplo, lo que un colegio hace y que parece muy bueno está al servicio de los otros colegios...”. (Sostenedor). 
Finalmente, el estudio confirma la literatura que sostiene que con una red muy densa de escuelas a cargo, se torna difícil prestar un apoyo técnico adecuado y se complejiza la gestión; un 89,5\% de los sostenedores que tienen 1 escuela a cargo dice contar con un equipo técnico para involucrarse directamente con el trabajo de los profesores. Quienes tienen entre 2 y 20 escuelas a cargo, un $85,1 \%$ dice contar con equipo técnico y quienes tienen entre 21 y 50 a cargo, solo un $60 \%$ dice contar con equipo técnico. Complementariamente, la literatura muestra que a mayor densidad, mayor complejidad administrativa y mayor dificultad para mantener coherencia en los mensajes a las escuelas.

\section{CONCLUSIONES Y DISCUSIÓN DE RESULTADOS}

Los resultados sobre el cumplimiento de metas SEP evidencian que, si bien el porcentaje de escuelas que cumple la meta no es muy alto $(17,6 \%)$, este número es positivo en términos de logro, considerando que el nivel de exigencias para esta primera cohorte de escuelas fue elevado. Por otra parte, e independientemente de si logran la meta exigida, el 72,5\% de las escuelas logró subir sus puntajes SIMCE en 4 años, lo que denota un considerable esfuerzo y un logro importante de la mayor parte de las escuelas del primer grupo participante. Es importante notar que la política SEP fue disminuyendo el nivel de exigencias de las metas a plantear por las escuelas a lo largo de su implementación, hasta que en 2015 se declaró que estas serían de carácter optativo dependiendo del criterio de la comunidad educativa de cada escuela.

En cuanto al papel que juega el liderazgo de los directores en el cumplimiento de las metas de aprendizaje, la incidencia de la práctica "observa aula y retroalimenta a los docentes" da cuenta de un cambio en las funciones que históricamente han asumido los directores, los que ahora se involucran en los aspectos instruccionales, demuestran interés por los aspectos pedagógicos y se hacen presentes en el aula. Si bien esta nueva orientación de las prácticas directivas favorecen el cumplimiento de las metas de aprendizaje, es importante considerar que este mayor foco en lo instruccional estaría produciendo una duplicación de funciones entre directores y jefes técnicos. Al respecto, la recomendación para la política que se desprende de los resultados es incorporar el concepto de liderazgo distribuido, según el cual los distintos líderes comparten un objetivo común, pero cumplen roles diferentes según las funciones que puedan desempeñar con mayor eficiencia, dado su rol formal o sus competencias profesionales y/o personales (Leithwood \& Mascall, 2008).

En cuanto a las prácticas de liderazgo de los sostenedores, la asociación negativa entre la centralización de las decisiones, el control sobre los recursos SEP y el espacio limitado para la gestión de los PM entrega una evidencia valiosa para la identificación de las funciones de un sostenedor efectivo. A partir de los resultados obtenidos se pueden considerar algunos antecedentes para la discusión del Proyecto de Ley que crea el sistema de educación pública, que actualmente se encuentra en el Congreso Nacional.

En primer lugar, resultaría relevante explicitar las responsabilidades y funciones del sostenedor respecto de las escuelas bajo su administración — cualquiera sea la figura institucional responsable de la gestión de las escuelas que se proponga en el Proyecto de Ley- asegurando que estas últimas cuenten con espacios de autonomía y flexibilidad para la gestión de los PM. Al igual que en el caso de los directivos, se sugiere que la política entregue pautas que pongan foco y orienten el rol de los sostenedores como líderes intermedios y articuladores entre las políticas y las escuelas. 
En segundo lugar, la nueva política debiera preocuparse de exigir a sostenedores competencias específicas en gestión y liderazgo educativo que la mayoría de ellos no tiene. Sería recomendable la generación de un marco de competencias específico para líderes intermedios, el cual podría acompañarse de un plan de capacitación y perfeccionamiento para sostenedores y otros líderes intermedios.

En tercer lugar, los datos apuntan a la necesidad de limitar la densidad de las redes de escuelas bajo la responsabilidad de un mismo sostenedor. Los resultados aquí analizados muestran que una alta densidad de escuelas dificulta la realización de un buen trabajo de colaboración entre estas y complejiza los canales para la participación. Una red densa complejiza su administración, reduce el alcance del liderazgo instruccional y aumenta las demandas sobre el equipo técnico, impidiendo que se responda con calidad a las necesidades que pueden surgir desde las escuelas.

Finalmente, la política debiera asegurar la disponibilidad de equipos técnicos suficientes como para prestar apoyo a las escuelas. Al respecto, es importante delimitar, asimismo, el rol técnico que pueden prestar los sostenedores del apoyo técnico que pueden prestar las ATE a las escuelas.

\section{REFERENCIAS BIBLIOGRÁFICAS}

Ainscow, M., Muijs, D., \& West, M. (2006). Collaboration as a strategy for improving schools in challenging circumstances. Paper prepared for 'Improving Schools'. University of Manchester, 9(3), 192-202.

Anderson, S. (2003). The School District Role in Educational Change: A Review of the Literature. Research on the role of the district. Ontario: ICEC, Ontario Institute for studies in Education, University of Toronto.

Anderson, S. (2010). Liderazgo directivo: claves para una mejor escuela. Psicoperspectivas. Individuo y Sociedad, 9(2), 34-52.

Barber, M., \& Mourshed, M. (2008). How the world's best-performing school systems come out on top. London: Mckinsey \& Company.

Burch, P., \& Spillane, J. (2004). Leading from the Middle: Mid-Level District Staff and Instructional Improvement. Chicago: Cross-City Campaign for Urban School Reform.

Camburn, E., Rowan, B., \& Taylor, J. E. (2003). Distributed leadership in schools: the case of elementary schools adopting comprehensive school reform models. Educational Evaluation and Policy Analysis, 25(4), 347-373.

Carrasco, R., Pérez, M., \& Núñez, D. (2015). Hacia una distribución más equitativa de las oportunidades educativas: ¿cuál es el impacto de la política de Subvención Preferencial en el desempeño académico de los alumnos más vulnerables en Chile? Pensamiento Educativo, 52(1), 65-83.

Chapman, C., \& Harris, A. (2004). Improving schools in difficult and challenging contexts: Strategies for improvement. Educational Research, 46, 219-228.

Dillon, E. (2011). The road to autonomy: Can schools, district, and central offices find their way? Washington DC: Education sector.

Elacqua, G., Mosqueira U., \& Santos, H. (2009). La Toma de Decisiones de un Sostenedor: Análisis a partir de la Ley SEP. Foco Educación, CPCE / Expansiva UDP, 1-37. Recuperado de http:// www.expansiva.cl/media/en_foco_edu/documentos/23062009104841.pdf

Elmore, R., Abelmann, C., \& Fuhrman, S. (1996). The new accountability in state education reform: From process to performance. En H. Ladd (Ed.), Holding schools accountable: Performance- 
Estudios Pedagógicos XLIII, $\mathrm{N}^{\circ}$ 1: 87-106, 2017

LIDERAZGO PARA LA MEJORA EN ESCUELAS VULNERABLES: PRÁCTICAS ASOCIADAS AL CUMPLIMIENTO

DE METAS DE APRENDIZAJE

based reform in education (pp. 65-98). Washington, DC: The Brookings Institution.

Epstein, J., Galindo, C., \& Sheldon, S. (2011). Levels of leadership: Effects of District and School Leaders on the Quality of School Programs of Family and Community Involvement. Educational Administration Quarterly, 47(3), 462-495.

Espínola, V., \& Silva, M.E. (2009). Competencias del sostenedor para una efectiva gestión del mejoramiento educativo en el nivel local: Una propuesta. Foco Educación, CPCE / Expansiva UDP, 1-31. Recuperado de http://www.expansiva.cl/media/en_foco_edu/ documentos/11122009123954.pdf

Espínola, V., Chaparro, M.J., Fuenzalida, A., Silva, M.E., \& Zárate, G. (2010). Estructura Organizacional de la Administración Educativa Municipal para Asegurar la Efectividad del Apoyo y Seguimiento a Escuelas Vulnerables. Evidencias para políticas Públicas en Educación: Selección de Investigaciones del Segundo Concurso FONIDE.

Firestone, W., \& Martinez, M.C. (2007). Districts, Teacher Leaders, and Distributed Leadership: Changing Institutional Practice. Leadership and Policy in Schools, 6(1), 3-35.

Fullan, M. (2005). Turnaround leadership. The Educational Forum, 69, 174-181.

Fundación Chile. (2010). Modelo de Gestión para Sostenedores de Calidad. Recuperado de http:// www.gestionescolar.cl/gestion-de-sostenedores.html.

González, A., González, M., \& Galdames, S. (2015). El sostenedor como agente de cambio: el rol de los coordinadores técnicos en el apoyo a establecimientos municipales chilenos. Pensamiento Educativo, 52(1), 47-64.

Gronn, P. (2002). Distributed leadership as a unit of analysis. Leadership Quarterly, 13(4), 423-451.

Hadfield, M. (2005). Middle leaders and the nature of distributed leadership in networks. Paper presented at the Annual Meeting of the American Educational Research Association, Montreal, Canada.

Hallinger, P., \& Heck, R.H., (2010). Leadership for Learning: Does Collaborative Leadership Make a Difference in School Improvement? Educational Management Administration \& Leadership, 38(6), 654-678.

Harris, A. (2009). Distributed leadership: what we know. In A. Harris (Ed.), Distributed Leadership: Different Perspectives (pp. 11-21). Dordrecht: Springer,

Harris, A., \& Spillane, J. (2008). Distributed leadership through the looking glass. Management in Education, 22, 31-34.

Heikka, J., Waniganayake, M., \& Hujala, E. (2012). Contextualizing Distributed Leadership With in Early Childhood Education: Current Understandings, Research Evidence and future Challenges. Educational Management Administration \& Leadership, 41(1), 30-44.

Hightower, A.M. (2002). San Diego's Big Boom: District Bureaucracy Supports Culture of Learning. Center for the Study of Teaching and Policy. Report R-02-2, Seattle, W.A: University of Washington.

Honig, M., Copeland, M., Rainey, L., Lorton, J.A., \& Newton, M. (2010). Central Office Transformation for District-wide Teaching and Learning Improvement. Center for the Study of Teaching and Policy, University of Washington.

Johnson, P., \& Chrispeels, J.H. (2010). Linking the central office and its schools for reform. Educational Administration Quarterly, 46(5), 738-775.

Jacobson, S., Johnson, L., Giles, C., \& Ylimaki, R. (2005). Successful leadership in U.S. schools: Enabling principles, enabling schools. Journal of Educational Administration, 43(6), 607-618.

Klar, H.W., \& Brewer, C.A. (2013). Successful Leadership in High-Needs Schools: An Examination of Core Leadership Practices Enacted in Challenging Contexts. Educational Administration Quarterly, 49(5), 768-808.

Larraín, T. (2005). Criterios para mejorar la coordinación con los sostenedores de escuelas municipales focalizadas. Un aporte al debate desde el Programa de las 900 Escuelas. Santiago, Chile: MINEDUC. 
Laso, M. del P., Albarrán, M., \& Carrasco, N. (2012). Gestión Educativa Municipal: Desafíos para una Educación de Calidad en Chile. Revista Iberoamericana de Estudios Municipales, 5, 95122. Recuperado de http://mapeal.cippec.org/wp-content/uploads/2014/05/Gesti\%C3\%B3nEducativa-Municipal.-Desaf\%C3\%ADos-para-una-educaci\%C3\%B3n-de-calidad-en-Chile.pdf

Leithwood, K. (2007). The 2005 Willower Family Lecture: Leadership according to the evidence. Leadership and Policy in Schools, 5(3), 177-202.

Leithwood, K., \& Jantzi, D. (2008). Linking leadership to student learning: The contributions of leader efficacy. Educational Administration Quarterly, 44(4), 496-528.

Leithwood, K., \& Mascall, B. (2008). Collective Leadership Effects on Student Achievement. Educational Administration Quarterly, 44(4), 529-561.

Leithwood, K., \& Riehl, C. (2005). What we know about successful school leadership. In W. Firestone, \& C. Riehl (Eds.), A new agenda: Directions for research on educational leadership (pp. 22-47). New York: Teachers College Press.

Leithwood, K., \& Strauss, T. (2008). Learning about leadership from school turnaround efforts in Ontario. Toronto: Canadian Education association.

Leithwood, K., Harris, A., \& Hopkins, D. (2008). Seven strong claims about successful school leadership. School Leadership and Management, 28(1), 27-42.

Leithwood, K., Harris, A., \& Strauss, T. (2010). Leading school turnaround: How successful leaders transform low-performing schools. San Francisco: Jossey-Bass.

Leithwood, K., Louis, K., Anderson, S., \& Wahlstrom, K. (2004). How leadership influences student learning. Review of research. University of Minnesota and University of Toronto. Commissioned by The Wallace Foundation.

Leithwood, K., Day, C., Sammons, P., Harris, A., \& Hopkins, D. (2006). Successful School Leadership: What It Is and How It Influences Pupil Learning. National College for School Leadership, Research Report $\mathrm{N}^{\circ} 800$.

Louis, K.S., Leithwood, K., Wahlstrom, K.L., \& Anderson, S.E. (2010). Learning from leadership: Investigating the links to improved student learning. New York: Center for Applied Research and Educational Improvement, University of Minnesota.

MacBeath, J. (2005). Leadership as distributed: a matter of practice. School Leadership and Management, 25(4), 349-366.

Mayrowetz, D. (2008). Making Sense of Distributed Leadership: Exploring the Multiple Usages of the Concept in the Field. Educational Administration Quarterly, 44(3), 424-435.

Ministerio de Educación de Chile. (2005). Marco para la Buena Dirección. Recuperado de http:// portales.mineduc.cl/usuarios/convivencia_escolar/doc/201103070155490.MINEDUC.Marco_ para_la_Buena_Direccion.pdf

Ministerio de Educación de Chile. (2008). Estudio sobre el Mejoramiento de la Gestión y la Calidad de la Educación Municipal. Santiago: Politeia, Soluciones Públicas.

Ministerio de Educación de Chile. (2012). Orientaciones Técnicas para la Elaboración del Plan de Mejoramiento Educativo. Recuperado de http://portales.mineduc.cl/usuarios/media/File/2012/ Noviembre/OrientacionesTEcnicas_PME_SEP_2013.pdf.

Mizala, A., \& Torche, F. (2013). ¿Logra la subvención escolar preferencial igualar los resultados educativos? Documento de Referencia $N^{\circ}$ 9. Santiago: Espacio Público.

Mourshed, M., Chijioke, C., \& Barber, M. (2010). How the World's Most Improved School Systems Keep Getting Better. New York, NY: McKinsey \& Company.

Muijs, D., West, M., \& Ainscow, M. (2010). Why network? Theoretical perspectives on networking. School Effectiveness and School Improvement, 21(1), 5-26.

Neumerski, C. (2012). Leading the Improvement of Instruction: Instructional Leadership in HighPoverty, Urban Schools (PhD Thesis). University of Michigan, Horace H. Rackham School of Graduate Studies.

Núñez, I., Weinstein, J., \& Muñoz, G. (2012). ¿Posición olvidada? Una mirada desde la normativa a 
la historia de la dirección escolar en Chile. En J. Weinstein y G. Muñoz (Eds.), ¿Qué sabemos sobre los directores de escuela en Chile? (pp. 371-396). Santiago: Fundación Chile-CEPPE.

OCDE. (2010). Pisa 2009 Results: What Students Know and Can Do: Student Performance in Reading, Mathematics and Science (Vol. I). París: OCDE.

Raczynski, D. (2012). Realidad de la Educación Municipal en Chile: ¿Liderazgo del sostenedor municipal? En J. Weinstein y G. Muñoz, (Eds.), ¿Qué sabemos sobre los directores de escuela en Chile? (pp. 181-217). Santiago: Fundación Chile-CEPPE.

Raczynski, D., \& Salinas, D. (2006). Gestión municipal de la educación: diagnóstico y líneas de propuesta. Santiago, Chile: Mineduc. Asesorías para el Desarrollo.

Raczynski, D., Muñoz, G., Weinstein, J., \& Pascual, J. (2013). Subvención Escolar Preferencial (SEP) en Chile: Un intento por equilibrar la macro y micro política escolar. REICE, 11(2), 164-193.

Robinson, V. M. J. (2007). School leadership and student outcomes: Identifying what works and why. Winmalee, NSW: Australian Council for Educational Leaders.

Román, M. (2007). El difícil cambio en los procesos de enseñanza aprendizaje en escuelas vulnerables chilenas. En J. Ansión y A.M. Villacorta (Eds.), Para comprender la Escuela Pública desde sus crisis y posibilidades (pp. 77-100). Lima: Fondo Editorial de la Pontificia Universidad Católica del Perú.

Sanders, M. (2012). Achieving Scale at the District Level: A Longitudinal Multiple Case Study of Partnership Reform. Educational Administration Quarterly, 48(1), 154-186.

Spillane, J.P. (1996). School districts matter: Local educational authorities and state instructional policy. Educational Policy, 10(1), 63-87.

Spillane, J.P., Camburn, E., \& Pareja A. (2007). Taking distributed perspective to the school principal's workday. Leadership and Policy in Schools, 6(1), 103-125.

Spillane, J.P., Halverson, R., \& Diamond, J. B. (2004). Towards a theory of leadership practice: A distributed perspective. Journal of Curriculum Studies, 36(1), 3-34.

Spillane, J.P., Camburn, E., Pustejovsky, J., Pareja A., \& Lewis, G. (2008). Talking a distributed perspective: Epistemological and methodological tradeoffs in operationalizing the leader-plus aspect. Journal of Educational Administration, 46(2), 189-213.

Umekubo, L., Chrispeels J., \& Daly, A. (2013). Estrechos lazos en un distrito descentralizado: estudio de caso de un distrito que mejora. Pensamiento Educativo, 50(2), 69-96.

Valenzuela, J.P., Villarroel, G., \& Villalobos, C. (2013). Ley de Subvención Escolar Preferencial (SEP): algunos resultados preliminares de su implementación, Pensamiento Educativo. Revista de Investigación Educacional Latinoamericana, 50(2), 113-131.

Walker, A., \& Ko, J. (2011). Principal leadership in an era of accountability: a perspective from the Hong Kong context. School Leadership \& Management, 31(4), 369-392.

Weinstein, J., Fuenzalida, A., \& Muñoz, G. (2010). La subvención preferencial: Desde una difícil instalación hacia su institucionalización. En S. Martinic y G. Elacqua (Eds.), ¿Fin de ciclo? Cambios en la gobernanza del sistema educativo (pp. 161-282). Santiago: Pontificia Universidad Católica de Chile/Unesco. 